\title{
Revisiting the Evaluation of the Effectiveness of Artemether-Lumefantrine Combination in the Treatment of Uncomplicated Malaria in Elele, a Malaria Endemic Area in Rivers State Nigeria
}

\author{
L. C. Chukwu ${ }^{*}$, P. U. Agbasi², P. C. Unekwe³ ${ }^{3}$ F. N. Oguwike ${ }^{4}$ \\ ${ }^{1}$ Department of Pharmacology, Chukwuemeka Odumegwu Ojukwu University, Igbariam, Nigeria \\ ${ }^{2}$ Department of Prosthetics and Orthotics, Federal University of Technology, Owerri, Nigeria \\ ${ }^{3}$ Department of Pharmacology, Nnamdi Azikiwe University, Awka, Nigeria \\ ${ }^{4}$ Department of Physiology, Chukwuemeka Odimegwu Ojukwu University, Igbariam, Nigeria \\ Email: *lenergy_chuks@yahoo.com
}

How to cite this paper: Chukwu, L.C., Agbasi, P.U., Unekwe, P.C. and Oguwike, F.N. (2019) Revisiting the Evaluation of the Effectiveness of Artemether-Lumefantrine Combination in the Treatment of Uncomplicated Malaria in Elele, a Malaria Endemic Area in Rivers State Nigeria. Journal of Biosciences and Medicines, 7, 59-72. https://doi.org/10.4236/jbm.2019.76005

Received: December 9, 2018

Accepted: June 14, 2019

Published: June 17, 2019

Copyright $\odot 2019$ by author(s) and Scientific Research Publishing Inc. This work is licensed under the Creative Commons Attribution International License (CC BY 4.0).

http://creativecommons.org/licenses/by/4.0/ (c) (i) Open Access

\begin{abstract}
Background: The World Health Organization adopted Artenisinin based combination therapy (ACT) for the treatment of uncomplicated malaria in endemic regions. The efficacy of ACT in malaria treatment must have prompted this choice. There's need to protect the ACT from plasmodial resistance. Hence, clinical scaling up of ACT program is needed. This entails continued assessment, monitoring and evaluation of the effectiveness of component drugs in endemic areas. Purpose: This study revisited the evaluation of the effectiveness of artemether-lumefantrine combination (ALC) in the treatment of uncomplicated malaria in Elele, Nigeria. Method: The study was conducted in Elele, a malaria endemic area in Rivers State, Nigeria. This was a facility based descriptive, cross sectional study at Madonna University Teaching Hospital (MUTH) Elele using simple sampling technique. Qualitative and quantitative data were collected. 100 patients who consented to the study were recruited in the outpatient clinic using semi structured questionnaires as part of study instrument. Inclusion criteria were having a body temperature of $\geq 37.5^{\circ} \mathrm{C}$, symptoms of malaria, positive parasitemia, non ingestion of antimalarial in the past 2 weeks, etc. MUTH ethics committee gave ethical clearance. Patient recruitment following consent commenced with their symptoms and signs recorded at presentation while disappearance of the same was determined following drug ingestion on days $2 / 4 / 6 / 8 / 10$ and 14. The data gotten was analyzed by tallying the responses to get the frequencies using SPSS 16.0 version and Microsoft excel tools. The student t-test was used to calculate the P-value, values $<0.05$ was considered statistically signif-
\end{abstract}


icant. Results: Most of the patients knew mosquitoes as the mode of malaria transmission (70\%). The ACT is known and used by these patients: artesunate/amodiaquine: 41 (41\%); artesunate/lumefantrine: 40 (40\%); artesunate/sulphadoxine-pyrimethamine: 38(38\%); artesunate/mefloquine: 20 (20\%). Fever was the predominant presenting symptom, $92 \%$ followed by body weakness $(90 \%)$; headache $(85 \%)$; malaise $(80 \%)$; loss of appetite $(80 \%)$; nausea (72\%); vomiting (70\%); abdominal pains (50\%). Others were: pallor (30\%); hepatomegally (20\%); splenomegally (20\%); chills (20\%); rigor (20\%). By day 10 of therapy, fever, vomiting and abdominal pains had disappeared in all patients, some patients still had mild: body weakness $40 \%$; headache $2 \%$; malaise $24 \%$; loss of appetite $20 \%$ and nausea $10 \%$. Day 14 recorded no symptoms in all patients. There was effective clinical response (ECR) by day 14 . Conclusion: This study hereby reaffirms the efficacy and effectiveness of Artemether-lumefantrine Combination in the treatment of uncomplicated malaria in Elele, Nigeria. The need to fill the existing knowledge gap of monitoring and evaluation of ACT in rural endemic areas has been done by this study.

\section{Keywords}

Uncomplicated Malaria, Fever, Effectiveness, Artemisinin Based

Combination, Elele

\section{Introduction}

Malaria is an infection of public health importance caused by the parasite of the genus Plasmodium. The species of the parasite causing human malaria in Nigeria are Plasmodium falciparum, $P$. vivax, $P$. ovale, $P$. malariae and $P$. knowlesi. $P$. falciparum is the commonest, implicated in about $97 \%$ cases of uncomplicated malaria. It is also the species responsible for most of the severe forms of the disease in Nigeria [1].

According to the World Malaria Report 2011, estimated 216 million cases of clinical malaria occurred in 2010 resulting in about 655,000 deaths across the globe. Of these deaths, $91 \%$ occurred in Africa [2].

About 2.2 billion people are exposed to malaria every year out of which 300 to 500 million develop the disease. Unfortunately, there were 247 million cases of malaria in 2006, causing nearly 1 million deaths, mostly among African children [3].

As a major public health problem in Nigeria malaria affects most pregnant women and children under the age of five. It impacts negatively on our economy with about 132 billion Naira loss to treatment and loss of man-hours. African leader's commitment to fighting malaria gave birth to a commendable launching of the Roll Back Malaria initiative on April 25, 2000. One of the key strategies to malaria control is effective case management. In 2005, the National Malaria Treatment Policy recommended the use of Artemisinin based Combination 
Therapy. These medicines still remain the most efficacious antimalarial treatments available [1]. Continuous monitoring and evaluation of this regimen are essential to maintaining its effectiveness. This knowledge gap needs to be filled.

Unfortunately, the increasing prevalence of antimalarial drug resistance is a major threat to malaria control globally, especially in resource poor countries like Liberia. The World Health Organization (WHO) has recommended Artemisinin-based combination therapy for the treatment of uncomplicated malaria in endemic countries. The rationale for the combination therapy is to improve the cure rate of malaria infections responding inadequately to monotherapy and possibly to prevent or delay the emergence of resistance strains [4].

It was exactly in April 2001, that the WHO recommended the use of artemisinin-based combination therapies (ACTs) for the treatment of uncomplicated malaria as well as ensured the highest cure rates and had the potential to reduce the spread of drug resistance [5].

Artemether-lumefantrine therapy combines the benefits of a rapid short acting schizonticidal effect of artemether with a slower but longer acting schizonticidal effect of lumefantrine, a highly lipophilic aryl amino alcohol [6] [7] [8].

It has been noted that a 3-day ACT's treatment course exposes 2 asexual cycles thereby reducing the number of malaria parasites in the body by approximately one hundred million-fold. An important bonus of the artemisinin compounds is their gametocytocidal activity which helps reduce transmissibility as well as decrease malaria incidence in low-transmission settings [9] [10].

To this end, Abdul and co concluded that to protect drug efficacy of AL, careful periodic monitoring of Artemether-lumefantrine combination therapy is essential to ensure pharmacologic effectiveness and safety in the management of uncomplicated falciparum malaria in Liberia and could make a substantial contribution to malaria control generally [8]. This monitoring is lacking in most endemic regions creating a knowledge gap that need to be filled.

\section{Malaria Symptoms as Tool for Disease Monitoring}

Malaria has varied non-specific symptoms including fever, chills, headache, muscular aching, weakness, vomiting, cough, diarrhea and abdominal pain. Other symptoms related to organ dysfunction may supervene, such as acute renal failure, pulmonary oedema, generalized convulsions, circulatory collapse, followed by coma and death. The initial symptoms can be mild, may not easily be linked to malaria [11].

Despite the non-specific nature of these symptoms, they are the basis for the initial clinical diagnosis of malaria disease. Laboratory diagnosis comes later as confirmatory.

Many studies have reported disparities in the mean fever clearance time in malaria infected subjects. Abdul et al. in Liberia reported 19 hours in Africans as compared to about 28 hours in Asians. This duration is much less than the re- 
ports of 26 to 72 hours in other international studies assessing the same Arthermeter-Lumefantrine efficacy. The noted disparities in the two races above may be due to the partial immunity of individual's resident in Africa resulting in early fever clearance [12] [13].

However, it is recommended that individuals who experiences fever should immediately inform medical personnel for diagnosis and effective treatment. This may also hold in the case of the possible exposure to malaria infection. Falciparum malaria may be fatal if treatment is delayed beyond $24 \mathrm{~h}$ after the onset of clinical symptoms [11].

In a study to addresses the therapeutic efficacy of ALC for the treatment of $P$. falciparum malaria in southwestern Ethiopia, Seleshi and co noted that monitoring drug efficacy in a 2-year interval is an important tool for establishing rational anti-malarial drug policies [14]. In that study, 74 (83.1\%) of the study subjects achieved fever clearance by day 1 , but $5(5.6 \%)$ still had fever at day 2 . All study subjects had no fever by day 3 .

The study further stated that, ALC has been highly effective in the treatment of uncomplicated $P$. falciparum malaria and reducing gametocyte carriage in southwestern Ethiopia years after its introduction in Ethiopia [14].

Our duty in this research is to reappraise the need for drug monitoring for effectiveness using symptoms clearance following Arthermeter-Lumefantrine combination therapy in the treatment of uncomplicated malaria in Elele, Rivers State Nigeria. Elele is a high malaria endemic area and its geographical map is represented in Figure 2 [15].

\section{Material and Methods}

\subsection{Study Area}

The study was conducted in Elele, a heavy malaria endemic area in Rivers State, South South Nigeria. This was a facility based descriptive, cross sectional study carried out at Madonna University Teaching Hospital (MUTH) Elele using simple sampling technique. Elele is a hyperendemic area for malaria infection. The climate could be warm with average temperature and heavy rainfall during its season. Vegetation is made up of thick forests which are annually and selectively deforested to serve as farm lands. Vegetation is reduced in the semi urban parts, the Elele town. The hydrographic network is fairly developed with many rivers, streams, swamps and lakes. The economy thrives mainly on fishing, agriculture and artisan business while the major means of transportation is the motorcycle riders popularly known as "Okada". Madonna University is a high institution with a tertiary Hospital serving the Elele populace. The map of the geographical location of Elele Nigeria, the study area is shown in Figure 2 [15].

\subsection{Study Population}

The study population consisted of patients made up of both sexes presenting with symptoms and signs suggestive of uncomplicated malaria infection and 
following adequate counseling, consented to participate in the study as well as signed the informed consent forms. The recruited candidates had ancillary body temperature of $\geq 37.5^{\circ} \mathrm{C}$, symptoms and signs suggestive of uncomplicated malaria with positive plasmodial parasitaemia, ability to take oral medication, non ingestion of any antimalarial 2 weeks prior to presentation, at least 15 years of age and resident close to the study area to encourage follow-up. Patients with a history of allergy to any of the component drugs and those with chronic diseases such as diabetes and high blood pressure or with immediate life-threatening medical conditions or have used any component drugs in the past 2 weeks were excluded from the study. A total of 100 malaria confirmed patients both men and women were enrolled for the study.

\subsection{Ethical Considerations}

Ethical clearance for this study was applied for and issued by the Ethics Committee of the Madonna University Teaching Hospital, Elele after approval of the protocol of this study. Prospective patients were approached during their clinic visits on daily basis. The aims and objectives of the study were explained to them in the language they understood best (Igbo, English or Broken languages), and their questions and curiosities were duly entertained to their satisfactions. Only volunteer patients were allowed to sign the informed consent forms. Patients found to have co morbidities were referred to the appropriate medical specialties for proper evaluation and management. Refusal to participate did not warrant denial of medical services neither did participation encore any added costs instead the medication was provided free of charge by the principal investigators.

\subsection{Date Collection, Questionnaire Completion and Symptoms Assessment}

A well designed questionnaire was administered to the participants enabling the documentation of their ages, sociodermographic characteristics, awareness of malaria, knowledge of modes of malaria transmission, types of antimalarial drugs known and various types of ACT. The patient's symptoms and signs at presentation were assessed and documented while laboratory tests for malaria parasites (MP tests) were carried out using patients blood samples to confirm the clinical diagnosis of malaria. Symptoms disappearance was determined in the patients taking the above regimen on days $2,4,6,8,10$, and 14 .

\section{Statistical Analysis}

The data gotten was analyzed, calculated to give the frequencies using SPSS 16.0 full version and Microsoft excel packages. The student $t$-test was used to calculate the $\mathrm{P}$-value and values of $<0.05$ was considered statistically significant.

\section{Results}

A total of 100 malaria confirmed patients both men and women were enrolled 
for the study which revisited the evaluation of the effectiveness of artemether-lumefantrine (ALC) combination in the treatment of uncomplicated malaria in Elele, Nigeria.

The awareness of the patients to malaria infection was noticed in 100 (100)\% of the participating patients (as shown in Table 1).

The knowledge of the participants about the modes of transmission of malaria infection was $70 \%$. A total of 70 patients identified mosquito bite as the mode of transmission. 10 patients (10\%) each of the participants identified cold and excessive fatty food as the mode of malaria transmission. Hunger and dirty environment were identified by $7 \%$ and $3 \%$ of the participating patients respectively (as shown in Table 2).

From the study, the participant's knowledge of antimalarial drugs which were as follows: Chloroquine 100\%; fansidar 98\%; quinine $88 \%$; artesunate $62 \%$; artesunate-amodiaquine $41 \%$ and artemether-lumefantrine $40 \%$ (as shown in Table 3 ).

While assessing the awareness of the participating patients to Artemisinin-based combination (ACT) antimalarial drugs it was found that, those aware of ACT's were $50 \%$ while those not aware were also $50 \%$ (as shown in Table 4 ).

The types of ACT's known among the patients and used in the past were as follows: Artesunate (AS)-amodiaquine (AQ) $=41 \%$; Artemether (AM)-lumefantrine

Table 1. Awareness of malaria infection among the participating patients.

\begin{tabular}{ccc}
\hline Awareness of malaria & No $=100$ & $\%$ \\
\hline Aware of malaria & 100 & 100 \\
Not aware of malaria & 0 & 0 \\
\hline
\end{tabular}

Table 2. The knowledge of mode of transmission of malaria among the participating patients.

\begin{tabular}{ccc}
\hline Mode of transmission & $\mathrm{N}=100$ & Percentage (\%) \\
\hline Mosquito bite & 70 & 70 \\
Cold & 10 & 10 \\
Excessive fatty food & 10 & 10 \\
Hunger & 7 & 7 \\
Dirty environment & 3 & 3 \\
\hline
\end{tabular}

Table 3. Participants knowledge of antimalarial drugs.

\begin{tabular}{ccc}
\hline Antimalarial & $\mathrm{N}=100$ & $\%$ \\
\hline Chloroquine & 100 & 100 \\
Fansidar & 98 & 98 \\
Quinine & 88 & 88 \\
Artesunuate & 62 & 62 \\
Artesunuate/Amodiaquine & 41 & 41 \\
Artesunuate/Lumefantrine & 40 & 40
\end{tabular}


$(\mathrm{LU})=40 \%$, Artesunuate (AS)-Sulfadoxine/Pyrimethamine (SP) $=38 \%$, Artesunuate (AS)-Mefloquine (MF) $=20 \%$ (see Table 5).

The pattern of symptoms the patients presented with on day 0 were as follows: fever $92 \%$; body weakness $90 \%$; headache $85 \%$; malaise $80 \%$; loss of appetite 80\%; nausea 72\%; vomiting 70\%; abdominal pains 50\% (as shown in Figure 1).

The pattern of symptoms the patients presented with on day 0 was presented in a table format for further clarification. Fever $92 \%$; body weakness $90 \%$; headache $85 \%$; malaise $80 \%$; loss of appetite $80 \%$; nausea $72 \%$, vomiting $70 \%$ and abdominal pains $50 \%$. Others (not shown) were pallor $30 \%$; hepatomegaly and splenomegaly $20 \%$ each; while chills and rigor were also $20 \%$ each (as shown in Table 6).

The interval between the disappearance of the presenting symptoms and commencement of treatment showed that by day 10 of therapy, fever, vomiting and abdominal pains had disappeared in all the 100 patients, some patients were still complaining of mild: body weakness $40 \%$; headache $2 \%$; malaise $24 \%$; loss of appetite $20 \%$ and nausea $10 \%$. By day 14 , no symptoms were reported in any of the 100 patients. There was Effective clinical response (ECR) by day 14 of therapy (as shown in Table 7).

Table 4. Awareness of artemisinin-based combination therapy (ACT) among the patients.

\begin{tabular}{ccc}
\hline Awareness of ACT's & $\mathrm{N}=100$ & $\%$ \\
\hline Aware of ACT'S & 50 & 50 \\
Not aware of ACT's & 50 & 50 \\
\hline
\end{tabular}

Table 5. Types of artemisinin-based combination therapy (ACT) known among the patients and used in the past.

\begin{tabular}{ccc}
\hline ACT known \& used & No $=100$ & $\%$ \\
\hline AS \& AQ & 41 & 41 \\
AM \& LU & 40 & 40 \\
AS \& SP & 38 & 38 \\
AS \& MF & 20 & 20 \\
\hline
\end{tabular}

Table 6. Pattern of symptoms the patients presented with on day 0.

\begin{tabular}{ccc}
\hline Symptoms & Frequency $=100$ & Percentage (\%) \\
\hline Fever $\left(\geq 37.5^{\circ} \mathrm{C}\right)$ & 92 & 92 \\
Body weakness & 90 & 90 \\
Headache & 85 & 85 \\
Malaise & 80 & 80 \\
Loss of appetite & 80 & 80 \\
Nausea & 72 & 72 \\
Vomiting & 70 & 70 \\
Abdominal pains & 50 & 50 \\
\hline
\end{tabular}


Table 7. Interval between commencement of treatment and the disappearance of symptoms.

\begin{tabular}{cccccccc}
\hline \multirow{2}{*}{ Symptoms } & \multicolumn{7}{c}{ Days } \\
\cline { 2 - 7 } & 0 & 2 & 4 & 6 & 8 & 10 & 14 \\
\hline Fever $(\mathrm{n}=92)$ & $92(100.0)$ & $52(56.5)(\mathrm{p}<0.05)$ & $10(10.8)$ & $5(5.4)$ & $2(2.1)$ & $0(0.0)$ & $0(0.0)$ \\
Body weakness $(\mathrm{n}=90)$ & $90(100.0)$ & $90(100.0)$ & $80(88.9)(\mathrm{p}<0.05)$ & $60(66.7)$ & $52(57.7)$ & $40(44.4)$ & $0(0.0)$ \\
Headache $(\mathrm{n}=85)$ & $85(100.0)$ & $70(82.4)(\mathrm{p}<0.05)$ & $62(72.9)$ & $20(23.5)$ & $15(17.6)$ & $2(2.4)$ & $0(0.0)$ \\
Malaise $(\mathrm{n}=80)$ & $80(100.0)$ & $70(87.5)(\mathrm{p}<0.05)$ & $60(75.0)$ & $42(52.5)$ & $36(45.0)$ & $24(30.0)$ & $0(0.0)$ \\
Loss of appetite $(\mathrm{n}=80)$ & $80(100.0)$ & $73(91.3)$ & $60(75.0)(\mathrm{p}<0.05)$ & $56(70.0)$ & $40(50.0)$ & $20(25.0)$ & $0(0.0)$ \\
Nausea $(\mathrm{n}=72)$ & $72(100.0)$ & $60(83.3)(\mathrm{p}<0.05)$ & $52(72.2)$ & $30(41.7)$ & $15(20.8)$ & $10(13.8)$ & $0(0.0)$ \\
Vomiting $(\mathrm{n}=70)$ & $70(100.0)$ & $36(51.4)(\mathrm{p}<0.05)$ & $22(31.4)$ & $14(20.0)$ & $2(2.9)$ & $0(0.0)$ & $0(0.0)$ \\
Abdominal pains $(\mathrm{n}=50)$ & $50(100.0)$ & $22(44.0)(\mathrm{p}<0.05)$ & $10(20.0)$ & $5(10.0)$ & $1(2.0)$ & $0(0.0)$ & $0(0.0)$ \\
\hline
\end{tabular}

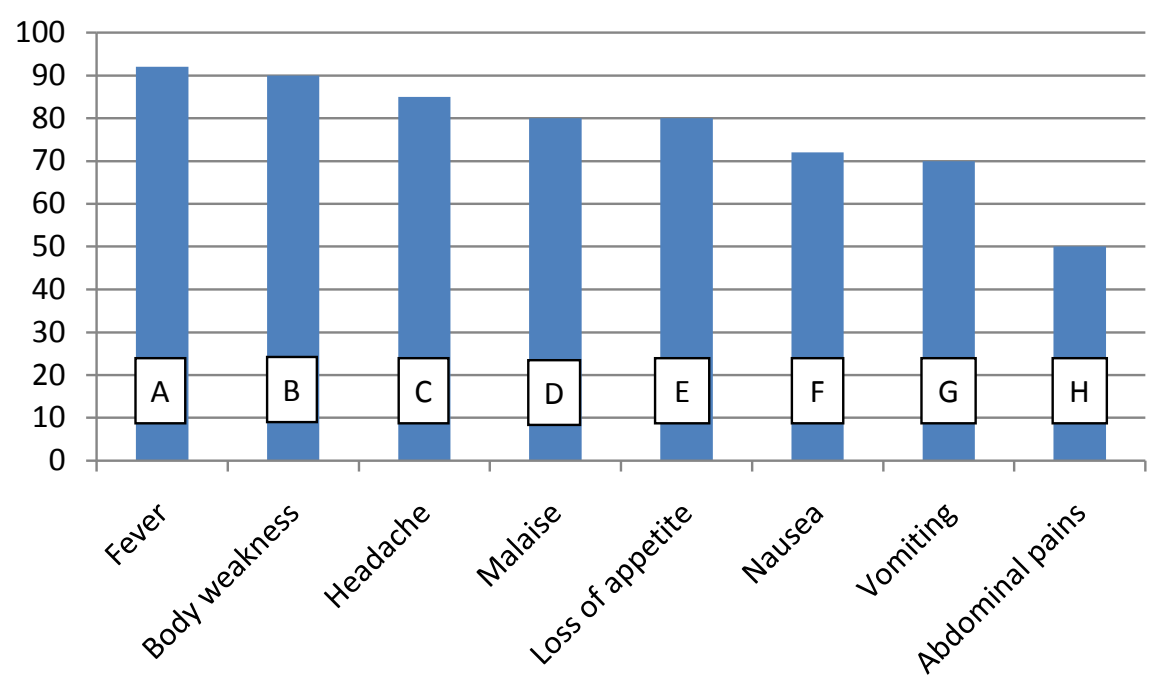

Figure 1. Pattern of Symptoms the Patients Presented With On Day 0. The symptoms are directed to their respective bars: KEY: $\mathrm{A}=$ Fever; $\mathrm{B}=$ Body weakness; $\mathrm{C}=$ Headache; $\mathrm{D}=$ Malaise; $\mathrm{E}=$ Loss of appetite; $\mathrm{F}=$ Nausea; $\mathrm{G}=$ Vomiting; $\mathrm{H}=$ Abdominal pains.

\section{Discussion}

This in-vivo study has re-evaluated the effectiveness of artemether-lumefantrine combination in the treatment of uncomplicated malaria in Elele, Nigeria. Although many studies have been conducted in different parts of the world on this topic, to our knowledge this is the only study which enrolled otherwise sick malaria diagnosed patients of both sexes irrespective of races and from 15 years of age. Perhaps also, it is the first study to have analyzed artemether lumefantrine combination effectiveness in this part of the world.

Awareness of the patients on malaria infection was very high as $100(100) \%$ of the patients were aware of malaria infection. Their knowledge of malaria infection compares to the outcome of a study carried out in the rural communities of Aliero, in Northern Nigeria in which 187 out of 200 respondents (93.5\%) admitted been aware of malaria infection [16]. 
A significant percentage of our participants demonstrated the correct knowledge of the mode of transmission of malaria infection 70 (70\%) by identifying mosquito bite as the mode of malaria transmission, In our study, only $10 \%$ each identified cold and excessive fatty food as the mode of transmission. Hunger and dirty environment were identified by $7 \%$ and $3 \%$ of the patients respectively. Their knowledge of mode of malaria transmission is far better than obtained in the above study where only $22(11.8 \%)$ respondents correctly stated that mosquitoe bite was the mode of malaria transmission [16]. However, our finding was consistent with another study in which $98.2 \%$ of the respondents reported mosquito bite as the cause of malaria. Though, in this quoted study, it wasn't well stated whether it referred to the exact malaria cause or its transmission mode [17]. Still in another study, there was a comparable knowledge of mode of transmission, $66 \%$ of the study community related the mode of transmission to the bite of infective mosquitoes though only $43.7 \%$ of them believed that malaria could be transmitted from person to person through the bite of mosquitoes [18].

From our study, we found out that the participants knowledge of antimalarial drugs which were as follows; Chloroquine 100\%; fansidar 98\%; quinine $88 \%$; artesunate $62 \%$; artesunate-amodiaquine $41 \%$ and artemether-lumefantrine $40 \%$. However in another study by Dickson et al., it was reported that antimalarials commonly cited for malaria treatment were chloroquine $(26.1 \%)$ and nivaquine (14.6\%), analgesics; panadol (23\%), and paracetamol (12.3\%) including native drugs (6.3\%) [19]. The high knowledge of Chloroquine (100\%), fansidar (98\%) and quinine $(88 \%)$ in our study can be attributed to the fact that they were the foremost antimalarial drugs before the advent of Arthemisinin based therapies.

The finding above had revealed an existing gap in knowledge of arthemisinin combination therapies over seventeen years after WHO recommendation it for the treatment of uncomplicated malaria [6] [7] [8]. To close this gap studies like ours are needed for the continuous monitoring and evaluation of antimalarials especially ACT's with a view to bettering malaria case management and limiting the emergence of antimalaria drug resistance.

On the various ACT's known and used by this study community the following result was gotten: Artesunate (AS)-amodiaquine (AQ) = 41\%; Artemether (AM)-lumefantrine (LU) $=40 \%$, Artesunuate (AS)-Sulfadoxine/Pyrimethamine $(\mathrm{SP})=38 \%$, Artesunuate (AS)-Mefloquine $(\mathrm{MF})=20 \%$. The study by Wakgari in Ethiopia, identified modern anti malaria usage as follows: The use of modern drugs for malaria was high (92\%) including chloroquine (73.5\%) and Sulfadoxine-Pyremethamine (60.6\%). In that study, chloroquine was believed to be effective for the treatment of malaria by $59 \%$ of the respondents, while the rest responded otherwise, ineffective [18].

Several years after most malaria endemic countries adopted the use of ACT's for the treatment of uncomplicated malaria; it is appalling that knowledge and practice of this medication has not reached the desired ebb even among some primary health care providers creating another research gap for exploitation. The Nigerian National Malaria Treatment Policy recommended the use of Ar- 
temisinin-based Combination Therapy in 2005, 13 years now [1]. In 2005, Cameroon also adopted ACT's for malaria treatment, hence a change from chloroquine or amodiaquine monotherapy to artemisinin-based combination therapy (AS/AQ) [19]. While in Malawi Linda et al., reported that due to increased resistance to sulphadoxine-pyrimethamine (SP), the Ministry of Health, as in many sub-Saharan African countries, changed the malaria treatment policy to use of artemisinin-based combination therapy (ACT) in 2008 [20].

In another study, Nduka et al. reported that $78 \%$ of their respondents were conversant with the ACT's while $71 \%$ of them stock ACTs in their shops. These were Patent Medicine Vendors [21]. In the Malawian study above, most of the participants (95.7\%) knew at least one form of ACT, $67.4 \%$ reported that different forms of ACT have different characteristics, $77.3 \%$ reported that there are special formulations for children. The most commonly mentioned ACT was artemether-lumefantrine (AL), by $94.6 \%$ of the participants and $75.0 \%$ of the participants indicated that they prefer to prescribe AL. $73.9 \%$ of participants had ever received information on ACT. However, only $31.5 \%$ had received training on management of malaria using ACT. There were $71.7 \%$ respondents who had heard of ACT causing side effects. Only $25.0 \%$ of the participants had received training on how to report SAEs. Again this is another research limitation requiring another study on the need to further train the patent medicine vendors on ACT's use, prescription, side effects etc. [20].

A study of the pattern of symptoms our patients presented with on day 0 showed that Fever was the most predominant presenting symptom 92\%. It was closely followed by body weakness and headache at $90 \%$ and $85 \%$ respectively. Malaise $80 \%$; loss of appetite $80 \%$; nausea $72 \%$, vomiting $70 \%$ and abdominal pains $50 \%$. Others (not shown on Table 6) were pallor 30\%; hepatomegaly and splenomegaly $20 \%$ each; while chills and rigor were also $20 \%$ each. Most studies analyzing malaria symptoms report fever as comparable to ours. In Ethiopia it was reported that majority $(86.8 \%)$ of the respondents mentioned fever as a symptom of malaria [17]. Other reported symptoms: chills, headache, nausea/vomiting, loss of appetite and body/joint aches were pointed out by $77.3 \%$, $64.8 \%, 46.7 \%, 40.8 \%$ and $31.5 \%$ of the respondents respectively as possible symptoms of malaria in the Ethiopian study. In another Ethiopian study, reports of fever clearance showed that $74(83.1 \%)$ of the study subjects achieved fever clearance by day 1 , but $5(5.6 \%)$ still had fever at day 2 . All study subjects had no fever by day 3 [14]. In one Northern Nigerian study, the most commonly mentioned symptom was fever with shivering by 122 respondents $65.2 \%$ [16] while in a Liberian study on malaria patients of both Asian and African races, fever was present in all subjects (100\%) at the time of enrollment from. Other main symptoms were chills, vomiting and headache while Splenomegaly was the leading sign. Thrombocytopenia (less than $150 \times 109 / 1$ ) was present in $33 \%$ and $28 \%$ of subjects from Asian and African races respectively [8]. In Ethiopia, Fever, headaches, chills and shivering were the most frequently mentioned symptoms of malaria as reported $89.7 \%, 87.5 \%$ and $81.3 \%$ of the study subjects re- 
spectively [18].

Since the emergence of some known malaria symptoms (though non specific) are a major reason for most patients to seek medical help, the disappearance of such symptoms also may usher in renewed complete health on those individual. Our patients presented to us with symptoms and signs suggestive of malaria on day 0 as above. For this reason, laboratory diagnosis was done to confirm our preliminary clinical diagnosis of malaria infection before recruitment. The disappearance of these symptoms were thus: on day 10 of therapy, fever, vomiting and abdominal pains had disappeared in all the 100 patients, some patients were still complaining of mild: body weakness $40 \%$; headache $2 \%$; malaise $24 \%$; loss of appetite $20 \%$ and nausea $10 \%$. By day 14 , no symptoms were reported in any of the 100 patients. There was Effective clinical response (ECR) by day 14 of therapy.

A further statistical interpretation of the disappearance of malaria symptoms among our patients holds that by day 2 of therapy there was significant reduction of some symptoms: fever 52\% (56.5) ( $\mathrm{p}<0.05)$; headache 70\% (82.4) $(\mathrm{p}<$ $0.05)$; malaise $70 \%$ (87.5) ( $\mathrm{p}<0.05$ ), nausea 60\% (83.3) ( $<<0.05)$; vomiting $36 \%$ (51.4) ( $\mathrm{p}<0.05)$; abdominal pain $22 \%(44.0)(\mathrm{p}<0.05)$. Significant reduction of some symptoms could only be achieved on day 4: body weakness $80 \%(88.9)(\mathrm{p}<$ $0.05)$; loss of appetite $60 \%(75.0)(\mathrm{p}<0.05)$.

The relevance of $p$-value in this study has to do with ascertaining the points (particular day) at which a particular symptom disappearance achieved statistical significant difference, $\mathrm{p}<0.05$ when compared with its state at presentation on day 0 . The disappearance of fever, headache and malaise were noted to be to be statistically significant on day 2 in all the participants $(\mathrm{p}<0.05)$. By definition, the $\mathrm{P}$ value, or calculated probability, is the probability of finding the observed, or more extreme, results when the null hypothesis $\left(\mathrm{H}_{0}\right)$ of a study question is true - the definition of "extreme" depends on how the hypothesis is being tested. P is also described in terms of rejecting $\mathrm{H}_{0}$ when it is actually true; however, it is not a direct probability of this state. However, most authors refer to statistically significant as $\mathrm{P}<0.05$ and statistically highly significant as $\mathrm{P}<0.001$, that is less than one in a thousand chance of being wrong [22]. A study on efficacy and safety of artemether-lumefantrine in uncomplicated falciparum malaria in Liberia reported a mean fever clearance time in P. falciparum infected subjects of about 19 hours in Africans and 28 hours in Asians. The disparity in these two races may not be unconnected with a higher partial malaria immunity of individual's resident in Africa that resulted in the early fever clearance. Also the duration is much less than that observed in some of the international studies assessing AL efficacy reported as 26 to 72 hours [8] [23] [24]. From our study, on day 6 of therapy all the patients, $100(100 \%)$ declared that they were already cured (no major symptoms, full activity restored and already back to work and now healthy). This shows a $100 \%$ effectiveness/efficacy of the artemether/lumefantrine combination therapy in the treatment of uncomplicated malaria 


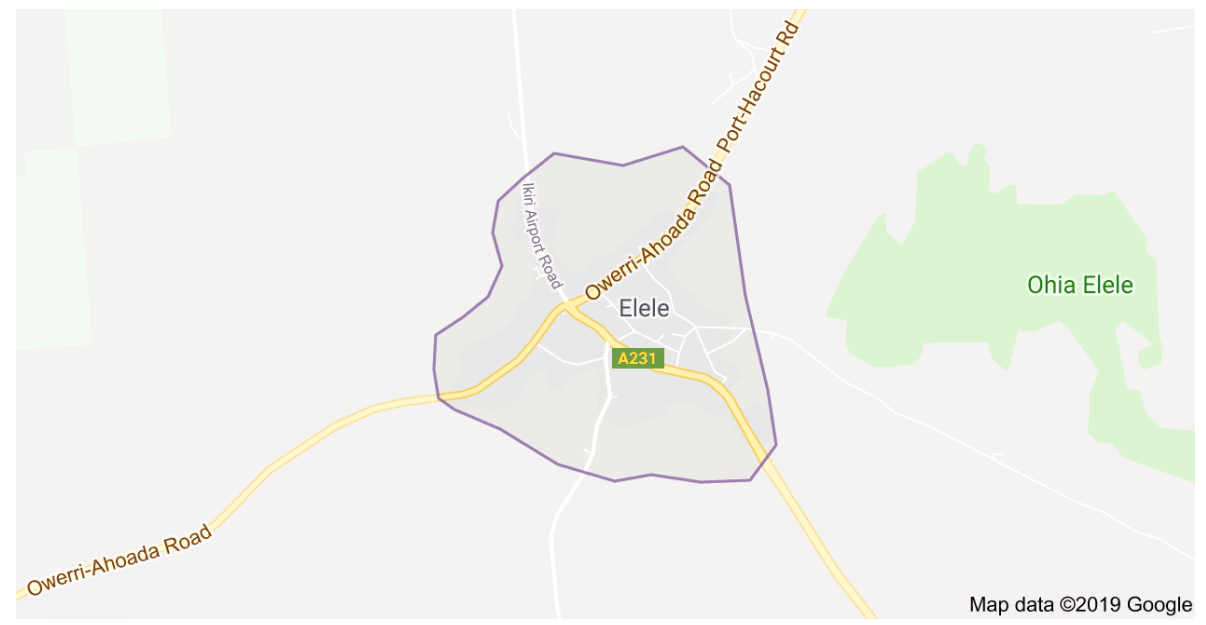

Figure 2. Map of the geographical location of Elele Nigeria, the study area. (Map data @2019 Google).

in Elele, in Rivers State, Nigeria. With the attainment of an Effective clinical response (ECR) by day 14 of therapy: we can deduce that artemether-lumefantrine combination therapy is $100 \%$ effective in the treatment of uncomplicated malaria in Elele Rivers State Nigeria, a high malaria endemic area. The geographical map/location of Elele in Nigeria, the study area is shown in Figure 2.

\section{Conclusion}

Artemether-lumefantrine combination therapy has been used in the effective management of uncomplicated malaria in Elele, Nigeria and is capable of making substantial contribution to malaria treatment and control programs. However, for this drug efficacy to be sustained as well as delay/prevent the emergence of resistance strains, continued periodic assessment, monitoring and evaluation of the efficacies and effectiveness of the component drugs especially in high malaria endemic areas need to be of utmost importance to health care providers.

\section{Acknowledgements}

We sincerely wish to thank our children and other family members of ours for their assistance in the course of this study. Our appreciation also goes to express our sincere thanks to our dear patients who volunteered to be part of this study. We also want to use this August medium to appreciate the doctors, midwives, nurses, lab technicians, home-visitors, cleaners, drivers, and logistical and administrative teams of Madonna University Teaching Hospital, Elele for all their assistance during the course of this study.

\section{Conflicts of Interest}

The authors declare that there were no conflicts of interests. 


\section{References}

[1] Federal Republic of Nigeria (2015) National Guideline for Diagnosis and Treatment of Malaria. Third Edition.

[2] UN Inter-Agency Group for Child Mortality Estimation (2012) Levels and Trends in Child Mortality: Report 2010. http://www.childinfo.org/files/Child

[3] Byakika-Kibwika, P., et al. (2010) Update on the Efficacy, Effectiveness and Safety of Artemether-Lumefantrine Combination Therapy for Treatment of Uncomplicated Malaria. Therapeutics and Clinical Risk Management, 6, 11-20. https://doi.org/10.2147/TCRM.S4483

[4] World Health Organization (2001) Antimalarial Drug Combination Therapy: Report of a WHO Technical Consultation. WHO/CDS/RBM/2001.35, WHO, Geneva.

[5] WHO (2006) Briefing on Malaria Treatment Guidelines and Artemisinin Monotherapies. Geneva.

[6] Shapiro, T.A. and Goldberg, D.E. (2006) Goldman and Gilman; the Pharmacological Basis of Therapeutics. 11th Edition, McGraw Hill, New York.

[7] Rosenthal, P.J. (2007) Antiprotozoal Drugs. In: Katzung, B., Ed., Basic and Clinical Pharmacology, 10th Edition, McGraw Hill, New York.

[8] Rasheed, A., Khan, S.M. and Awan, M.Y. (2011) Efficacy and Safety of Artemehter-Lumefantrine in Uncomplicated Falciparum Malaria in Liberia. Journal of Pakistan Medical Association, 61, 131-134.

[9] Price, R.N., Nosten, F., Luxemburger, C., ter Kuile, F., Paiphun, L., Chongsuphajaisiddhi, T. and White, N.J. (1996) The Effects of Artemisinin Derivatives on Malaria Transmissability. The Lancet, 347, 1654-1658. https://doi.org/10.1016/S0140-6736(96)91488-9

[10] Nosten, F. and White, N.J. (2007) Artemisinin-Based Combination Treatment of Falciparum Malaria. American Journal of Tropical Medicine and Hygiene, 77, 181-192.

[11] World Health Organization (2018) International Travel and Health.

[12] Falade, C.O., Ogunkunle, O.O., Dada-Adegbola, H.O., Falade, A.G., dePalacious, P.I., Hunt, P., et al. (2008) Evaluation of the Efficacy and Safety of Artemether-Lumefantrine in the Treatment of Acute Uncomplicated Plasmodium Falciparum Malaria in Nigerian Infants and Children. Malaria Journal, 7, 246. https://doi.org/10.1186/1475-2875-7-246

[13] Kobbe, R., Klein, P., Adjei, S., Amemasor, S., Thompson, W.N., Heidemann, H., et al. (2008) A Randomized Trial on Effectiveness of Artemether-Lumefantrine versus Artesunate plus Amodiaquine for Unsupervised Treatment of Uncomplicated Plasmodium falciparum Malaria in Ghanian Children. Malaria Journal, 7, 261. https://doi.org/10.1186/1475-2875-7-261

[14] Mekonnen, S.K., Medhin, G., Berhe, N., Clouse, R.M. and Aseffa, A. (2015) Efficacy of Artemether-Lumefantrine Therapy for the Treatment of Uncomplicated Plasmodium falciparum Malaria in Southwestern Ethiopia. Malaria Journal, 14, 317. https://doi.org/10.1186/s12936-015-0826-9

[15] Map Data @2019 Goggle.

[16] Singh, R., Musa, J., Singh, S. and Ebere, U.V. (2014) Knowledge, Attitude and Practices on Malaria among the Rural Communities in Aliero, Northern Nigeria. Journal of Family Medicine and Primary Care, 3, 39-41. https://doi.org/10.4103/2249-4863.130271 
[17] Astatkie, A. (2010) Knowledge and Practice of Malaria Prevention Methods among Residents of Arba Minch Town and Arba Minch Zuria District, Southern Ethiopia. Ethiopian Journal of Health and Medical Sciences of Jimma University, 20, 185-193. https://doi.org/10.4314/ejhs.v20i3.69448

[18] Wakgari, D., Ahmed, A. and Fikre, E. (2004) Knowledge, Attitude and Practice about Malaria, the Mosquito and Antimalarial Drugs in a Rural Community. Ethiopian Journal of Health Development, 17, 99-104.

https://doi.org/10.4314/ejhd.v17i2.9849

[19] Nsagha, D.S., Njunda, A.L., Kamga, H.L.F., Nsagha, S.M., Assob, J.C.N., et al. (2011) Knowledge and Practices Relating to Malaria in a Semi-Urban Area of Cameroon: Choices and Sources of Antimalarials, Self-Treatment and Resistance. The Pan African Medical Journal, 9, 8. https://doi.org/10.4314/pamj.v9i1.71180

[20] Kalilani-Phiri, L.V., Lungu, D. and Coghlan, R. (2011) Knowledge and Malaria Treatment Practices Using Artemisinin Combination Therapy (ACT) in Malawi: Survey of Health Professionals. Malaria Journal, 10, 279. https://doi.org/10.1186/1475-2875-10-279

[21] Nduka, S.O., Agbata, C.A., Eze, B.H., Ezeobi, I., Nwadukwe, C.C. and Uzodinma, S.U. (2013) Assessment of Artemisinin Based Combination Therapy Utilization among Patent Medicine Vendors in Some Rural Areas in Nigeria. International Journal of Collaborative Research on Internal Medicine and Public Health, 5, 619-629.

[22] https://www.statsdirect.com/help/basic/p value.htm

[23] Falade, C.O., Ogundele, A.O., Yusuf, B.O., Ademowo, O.G. and Ladipo, S.M. (2008) High Efficacy of Two Artemisinin Based Combinations (Artemether-Lumefantrine and Artesunate plus Amodiaquine) for Uncomplicated Malaria in Ibadan, Nigeria. Tropical Medicine \& International Health, 13, 635-643. https://doi.org/10.1111/j.1365-3156.2008.02043.x

[24] McGready, R., Tan, S.O., Ashley, E.A., Pimanpanarak, M., Viladpai-Nguen, J., Phaiphun, L., et al. (2008) A Randomized Controlled Trial of Artemether-Lumefantrine versus Artesunate for Uncomplicated Plasmodium falciparum Treatment in Pregnancy. PLOS Medicine, 5, e253. https://doi.org/10.1371/journal.pmed.0050253 